\title{
Diagnostic accuracy of (18) F-FDG PET/CT for the detection of peritoneal carcinomatosis of colorectal origins
}

\author{
Bullier $\mathrm{E}^{1}$, Descat $\mathrm{E}^{2}$, Bonichon $\mathrm{F}^{1}$, Picat $\mathrm{MQ}^{3}$, Bellera $\mathrm{C}^{3}$, Evrard $\mathrm{S}^{4}$ and Cazeau $\mathrm{AL}^{1, *}$ \\ ${ }^{1}$ Department of Nuclear Medicine, Institut Bergonié, 229 cours de l’Argonne, 33076 Bordeaux, France \\ ${ }^{2}$ Department of Radiology, Institut Bergonié, 229 cours de l'Argonne, 33076 Bordeaux, France \\ ${ }^{3}$ Clinical Research and Epidemiological Unit, Institut Bergonié, 229 cours de l'Argonne, 33076 Bordeaux Cedex, France \\ ${ }^{4}$ Department of Surgery, Institut Bergonié, 229 cours de l’Argonne, 33076 Bordeaux Cedex, France
}

\begin{abstract}
Purpose: to evaluate the diagnostic accuracy of (18)F-FDG positron emission tomography/ computerized tomography (PET/CT) for the detection of peritoneal carcinomatosis (PC) of colorectal cancer (CRC). Methods: one hundred and forty six eligible patients referred for a PET/CT to evaluate CRC at a single institution were included consecutively and retrospectively. After joint training on 20 similar patient files, two nuclear physicians reviewed the PET/CT scans blindly and independently using a method specifically designed for PC detection. The gold standard was either histological results from surgical exploration for resected patients ( $\mathrm{n}=65$ ) or clinical follow up defined as the 3-month CT follow up supplemented by one year of clinical information and CT monitoring for non-resected patients $(\mathrm{n}=81)$. As secondary objectives we evaluated the interobserver reliability between the two PET/CT readings and the diagnostic accuracy of a contemporary ceCT $(n=69)$ or CT component of the PET/CT $(n=77)$ reviewed by an independent radiologist. The extent of PC according to a simplified Sugarbaker index (PCI) was examined for the operated subgroup, when PCI was available from the surgeon and PET/CT ( $\mathrm{n}=12)$. Results: according to the gold standard, 35/146 patients had PC (including 19 of 65 patients with histological gold standard and 16 of 81 patients with clinical follow up gold standard). Sensitivity and specificity of PET/ CT were $88 \%$ and $96 \%$ respectively and positive and negative predictive values and accuracy, 88\%, 96\% and 94\% respectively. For CT alone, the corresponding values were: $68 \%, 92 \%, 72 \%, 90 \%$ and $86 \%$. The interobserver agreement for the detection of peritoneal carcinomatosis in PET/CT showed high agreement at 0.91 (Kappa). Median PCI was 3 [range: 1-13] when assessed by surgeons and 4 [range: 1-10] on PET/CT. Focal uptake was the most frequently observed sign on PET. Conclusions: PET/CT appears to be an accurate and reproducible test for PC diagnosis in colorectal cancer using an interpretation method specifically designed for PC detection. To confirm these results and to evaluate peritoneal carcinomatosis extension accurately, a prospective observational study using iodine contrast-enhanced PET/CT as a first imaging technique for pre-operative staging and potentially involving further independent reviewers remains to be undertaken.
\end{abstract}

Keywords: PET/CT; peritoneal carcinomatosis; colorectal cancer; diagnostic accuracy

\section{Introduction}

Treating a patient with colorectal metastatic disease requires checking three major targets of the cancer: the liver, the lung and the peritoneum. Liver metastases are the most treatable localisation and aggressive curative treatments including induction chemotherapy, two-stage procedures and intraoperative ablation are reported with promising results [1] even if the lesions are bilateral and numerous. Nevertheless, facing these complex situations, it is important to check other potential metastatic sites. If a very small and localised carcinomatosis can be resected in the same operative time or if there is only a limited number of small lung metastases (generally less than 3), the curative hepatic approach can be maintained.
Previously, when a colorectal cancer spread to the peritoneum, this was considered to be a terminal development with median survival around 5-12 months

*Corresponding author: Cazeau AL, Institut Bergonié, 229 cours de l'Argonne, 33076 Bordeaux Cedex, France, Tel.: +33 556333321 Fax: +33556333384 E-mail: a.cazeau@bordeuaux.unicancer.fr

Received 11 December 2012 Revised 22 January 2013 Accepted 28 January 2013 Published 5 February 2013

Citation: Bullier E, Descat E, Bonichon F, Picat MQ, Bellera C, Evrard S, Cazeau AL (2013) Diagnostic accuracy of (18)F-FDG PET/CT for the detection of peritoneal carcinomatosis of colorectal origins. J Cancer Res Ther 1: 46-53. doi:10.14312/2052-4994.2013-7

Copyright: (c) 2013 Bullier E, et al. This is an open-access article distributed under the terms of the Creative Commons Attribution License, which permits unrestricted use, distribution and reproduction in any medium, provided the original author and source are credited. 
after systemic chemotherapy [2]. If the peritoneum is the main metastatic site, surgical treatment based on cytoreductive surgery possibly associated with intraperitoneal hyperthermic chemotherapy, can improve prognosis with survival now reaching up to 60 months [3]. Nevertheless, a surgical procedure can be proposed only if the Sugarbaker index of peritoneal spreading (PCI) does not exceed 20/39. By analogy, a small number of liver metastases is not a contra-indication to resect the peritoneum [4, 5]. Consequently, it is very important that the pre-therapeutical evaluation of the disease be as precise as possible to be discussed in a multidisciplinary team meeting as completely different strategies can be decided upon depending on the information acquired. PCs are generally identified via surgical exploration of the abdominal cavity and specific assessment of disease extension should be made to evaluate resectability [6]. However, making a diagnosis of PC in a noninvasive way remains a major issue. While Computed Tomodensitometry (CT) is currently the reference testing method [3], fluorine-18 fluorodeoxyglucose (18F-FDG) Positron Emission Tomography (PET) appears to have an important role to play. Promising results highlight the use of 18F-FDG PET to evaluate PC extension pre-operatively $[7,8]$ although only a few reports have been published on the diagnostic performance of 18F-FDG PET/CT. Sensitivities reported have varied from unacceptably low (35\%) to $100 \%$ [7-14].

The principal objective of this study was to evaluate the diagnostic performance of 18F-FDG PET/CT for the detection of a PC of colorectal origin using as gold standard the histological results for resected patients or one year of clinical monitoring for non-resected patients. Common review and interpretation criteria were used to interpret PET/CT images and identify indicative signs of PC. In addition, PET/CT diagnostic accuracy was compared with diagnostic accuracy of CT alone read by an experimented radiologist, interobserver agreement was examined and indicative signs indicative of PC were fully explored, also the extent of PC with a simplified score.

\section{Materials and methods}

\section{Patients}

All adult patients who received an 18F-FDG PET/CT scan during care for histologically diagnosed colorectal carcinoma at our institution over 2004-2007 were included consecutively and retrospectively in this series. Databases at our institution contained 250 PET/CT scans obtained between 2004 and 2007, corresponding to 160 patients. Fourteen patients were excluded because of a lack of follow up data. If a patient had received more than one PET/CT scan, only the first was examined for diagnostic accuracy. One hundred and forty six scans for 146 patients are thus maintained for analyses. The PET/CT scans were obtained in one of three settings: relapse suspicion $(n=89)$, relapse examination $(n=57)$ or pre-operatively for primary colorectal cancer $(n=2)$. Institutional review board approval was obtained for this study. Informed patient consent was not required for these retrospective analyses.

\section{Clinical patient characteristics}

Median patient age was 63.5 years (min/max: 23-88 years) and $50.7 \%$ of patients were women. All patients had colorectal adenocarcinoma (126 non-mucinous, 20 mucinous) and no patients had appendiceal cancers. Tumour marker status was available for a subset of patients. Median CEA was 5.9 IU/L (range: 0.6-1650) $(\mathrm{n}=90)$ and median CA19.9 IU/L was 14 (range: 1-679.7) $(n=61)$. In terms of previous treatments received which potentially could change the FDG uptake, 124 patients received chemotherapy before PET/CT with a median delay of 7 months (min/max: 1-148 months). One hundred and forty four patients had previously received abdominal surgery before inclusion in the PET/CT study with a median delay of 14.5 months (min/max: 1-160 months).

\section{PET/CT procedure}

After at least $6 \mathrm{~h}$ of fasting and capillary glycaemia measurement (less than $2.0 \mathrm{~g} / \mathrm{L}$ ) we injected $5 \mathrm{MBq} / \mathrm{kg}$ of 18F-FDG intravenously. Median uptake delay was 70 min (54-161 min). Whole-body PET/CT images were obtained on a PET scanner Discovery ST (General Electric Medical System, Milwaukee, USA) in 3D mode, without septa, producing 47 slices over an approximately $150 \mathrm{~mm}$ axial field of view and a 3 min time of acquisition per step from the base of the skull to the base of the thighs. The imaging acquisition parameters were in-plane and axial resolution of 3.91 and $3.27 \mathrm{~mm}$ FWHM, respectively, inplane field of view of $600 \mathrm{~mm}, 128 \times 128$ pixels matrix. An image matrix of $256 \times 256$ pixels was used for iterative reconstruction (FORE+OSEM; subsets: 32; iterations: 5). CT transmission scans were acquired previously to the PET scanner for attenuation correction, without oral or intravenous contrast injection, with the constants of 140 $\mathrm{kV} / 80 \mathrm{mAs}$ and slices of $3.75 \mathrm{~mm}$.

\section{Gold standard}

The gold standard was the histology results from surgical exploration for all resected patients 65/146 (44.5\%). The surgery was carried out with a median delay of 2 months after PET/CT. For non-resected patients $(n=81$, $55.5 \%$ ), the gold standard was the radiologist's diagnosis made from the first planned CT scan at three months, supplemented by information from one year of clinical and CT monitoring. The radiologist's diagnosis was based on morphological criteria (peritoneal nodal tissue, fat infiltration, epiploic thickening, and ascites) and was thus verified and confirmed against the clinical-radiological information identified in the patient's follow up files over one year, notably any shrinkage of peritoneal lesion under chemotherapy during follow up was considered as PC. Possible PC diagnoses made by the radiologist were discarded if no suspect events were observed clinically or by CT scanner after one year of follow up without systemic therapy. 


\section{PET/CT imaging review and interpretation criteria}

Review of the PET/CT images in our series was performed independently by two nuclear physicians (ALC: 6 years of experience; EB: 4 years' experience) who were not aware of the histological results or of the radiologist's CT reading at three months. Each reviewer concluded with a judgment of presence or absence of PC. When the two readers did not agree, a consensus was established by a secondary joint reading. The consensus diagnosis was used for the statistical evaluation of PET/CT performance.

A preliminary joint study between the two readers on 20 PET/CT files selected from the colorectal PET scan obtained after study inclusion outside the scope of this study allowed the two readers to define common review and interpretation criteria. Examining the images was done systematically: first by visualising the maximum intensity projections MIP of the PET across $360^{\circ}$ and by triangulating on each visible intra-abdominal uptake, then on each series of images (PET alone, then CT alone, then PET/CT together). Each image series was visualised in the three conventional orthogonal cross-sections. Sagittal plan was specially observed for pelvic area and to highlight a straight line sign. Coronal plane was used to explore the peri-hepatic area.

\section{Signs indicating PC were}

Scintigraphic signs: focal uptake [13], diffuse peri-hepatic curvilinear uptake [11], sub parietal (epiploic) uptake or the presence of a straight line sign [15, 16]; and morphologic signs: peritoneal nodal tissue, fat infiltration, epiploic thickening, ascite [17]. The two reviewers looked for lesions across the whole of the peritoneal cavity with particularattention to thelevel of theleftand rightparacolic gutter, the peri-hepatic area, the pelvis and the omentum. A PC was diagnosed when evident morphologic anomalies were present, even when there were no scintigraphic anomalies or when evident scintigraphic anomalies were present even without morphologic anomalies on CT (for example, small-intestinal focal uptake).

Lymph node (well-differentiated nodule tissue close to vascular axes, mostly found in the ileocaecal region), post-operative inflammatory reactions (such as anterior peritoneal uptakes associated with linear longitudinal sub-cutaneous soft-tissue anomalies on aspect), nonhypermetabolicmesenteric panniculitisaspects, accessory spleens (well-limited peri-splenic nodular tissue) and colic or small-intestinal diffuse digestive uptake with no morphologic anomalies on fusion CT were not considered to be PCs. The two reviewers could conclude as "negative for PC", "probably negative", "probably positive", or "positive for PC".

\section{Extent of PC}

A simplified Sugarbaker index was calculated, keeping the area grading from 0 to 3 according to the size of the lesion ( 1 for a lesion $<5 \mathrm{~mm}, 3$ for a lesion $>5 \mathrm{~cm}$, and 2 for between $5 \mathrm{~mm}$ and $5 \mathrm{~cm}$ ). The involved areas are the abdominal cavity area (right and left hypochondria, epigastrium, right and left flanks, para-ombilical, right and left iliac fossae and pelvis), reducing the small bowel to only one area instead of 4 , which is more realistic for slice imagery.

\section{Review of recent CT images for comparison study}

Contemporary CT scans were reviewed blindly by a radiologist with digestive oncology experience to judge whether a PC was present or not. The CT images selected were radiologically-enhanced, ceCT $(n=69)$ and obtained less than three months before the PET/CT. For patients for whom no recent CT scan was available, the CT component (non-enhanced) from PET/CT was reviewed by the radiologist $(n=77)$. The radiologist's diagnosis was based on morphological criteria (peritoneal nodal tissue, fat infiltration, epiploic thickening, and ascites). The reviewer made one of four conclusions: "negative for PC", "probably negative", "probably positive", or "positive for PC".

\section{Statistical considerations}

For the statistical analyses, presence of PC was defined as "PC positive" or "probably PC" for each reader and PC absence was "negative for PC" and "probably negative". The diagnostic accuracy of the PC diagnosis by PET-CT was examined by the sensitivity and specificity, the positive (PPV) and negative predictive values (NPV), and the accuracy, which are described with their 95\% confidence intervals (CI). Diagnostic accuracy of the two gold standard components are compared, as are diagnostic accuracy of CT enhanced vs. non-enhanced, and CT vs. PET/CT. The interobserver agreement for the PC diagnosis by PET/ CT was evaluated by the Kappa coefficient, ranging from 0 (no agreement) to 1 (perfect agreement). The study of signs consisted in a descriptive analysis.

\section{Results}

The overall rate of PC in our series was 24.0\% (35/146). The incidence for patients with a histological gold standard was 19 of 65 patients (29.2\%), and for patients with CT follow up gold standard: 16 of 81 (19.8\%).

Diagnostic performances of PET/CT vs. gold standard in the detection of peritoneal carcinomatosis

The sensitivity of the PET/CT imaging for the detection of PC was $88.6 \%$ (95\% CI 83.4-93.8), specificity $96.4 \%$ (95\% CI 93.4-99.4), PPV 88.6\% (95\% CI 83.4-93.8), NPV 96.4\% (95\% CI 93.4-99.4) and accuracy 94.5\% (95\% CI 90.8-98.2) (Table 1). Given the slight difference in incidence rates observed according to type of gold standard, we performed secondary analyses for the diagnostic performance of PET/CT according to type of gold standard. The diagnostic performance of PET/CT was similar for the 65 patients with a surgical gold standard: 
sensitivity $89.5 \%$ (95\%CI 82.0-97.0), specificity $95.7 \%$ (95\%CI 90.8-100), PPV 89.5\% (95\% CI 82.0.-97.0), NPV 95.7\% (95\% CI 90.8-100) and accuracy 93.8\% (95\% CI 87.9-99.6) as for the 81 patients with CT follow up gold standard: Sensitivity 93.3\%, 95\%CI (68.1-99.8), specificity 98.5\%, 95\% CI (91.8-100.0), PPV 93.3\% (68.1\% - 99.8\%), NPV 98.5\% (91.8\%-100.0\%) and accuracy $97.5 \%$ (91.4\% - 99.7\%).

Table 1 Rates of detection of peritoneal carcinomatosis by PET/CT vs. gold standard

\begin{tabular}{|c|c|c|c|}
\hline \multicolumn{4}{|c|}{ Peritoneal carcinomatosis diagnosis after PET/CT imaging } \\
\hline \multirow{2}{*}{$\begin{array}{c}\text { Peritoneal } \\
\text { carcinomatosis } \\
\text { diagnosis after } \\
\text { PET/CT imaging }\end{array}$} & \multicolumn{2}{|c|}{ gold standard } & \multirow{2}{*}{$\begin{array}{c}\text { Total } \\
(N)\end{array}$} \\
\hline & $\begin{array}{l}\text { Positive } \\
\text { (N) }\end{array}$ & $\begin{array}{c}\text { Negative } \\
\text { (N) }\end{array}$ & \\
\hline Positive (N) & 31 & 4 & 35 \\
\hline Negative $(\mathrm{N})$ & 4 & 107 & 111 \\
\hline Total (N) & 35 & 111 & 146 \\
\hline
\end{tabular}

Diagnostic performance of CT vs. gold standard in the detection of peritoneal carcinomatosis

For comparison purposes, diagnostic accuracy of CT alone was calculated. Sixty-nine patients had a CT scan taken just before the PET/CT that was available for review with a maximum delay of 3 months and for the remaining 77 patients, the review was carried out on the CT of the PET/CT imaging. As demonstrated by the raw counts in Table 2, the sensitivity of the CT imaging overall for the detection of PC was 68.6\% (95\% CI 61.176.1), the specificity $92.9 \%$ (95\% CI 88.7-97.1), the PPV 72.7\% (95\% CI 65.5-79.9), the NPV 90.3\% (95\% CI 85.5-95.1) and the accuracy $86.3 \%$ (95\% CI 80.7-91.9). There were no differences observed between diagnostic accuracy of CT with or without contrast enhancement: CT with enhancement ( $\mathrm{n}=69$ ): Sensitivity 64.3\% 95\% CI (35.1\% - 87.2\%), specificity $90.9 \%$ (80.1\% - 97.0\%), PPV $64.3 \%$ (35.1\% - 87.2\%), NPV 90.9\% (80.1\% - 97.0\%) and accuracy $85.5 \%$ (75.0\% - 92.8\%), compared to without enhancement (from PET/CT) (n=77): Sensitivity: 70\%, 95\%CI $(45.7 \%-88.1 \%)$, specificity $93.0 \%(83.0 \%$ 98.1\%), PPV 77.8\% (52.4\% - 93.6\%), NPV 89.8\% (79.2\% - 96.2\%) and accuracy $87.0 \%$ (77.4\% ; 93.6\%).

Table 2 Rates of detection of peritoneal carcinomatosis by CT vs. gold standard

\begin{tabular}{lccc}
\hline \multicolumn{4}{c}{ Peritoneal carcinomatosis diagnosis after CT imaging } \\
\hline $\begin{array}{c}\text { Peritoneal } \\
\text { carcinomatosis } \\
\text { diagnosis after } \\
\text { CT imaging }\end{array}$ & $\begin{array}{c}\text { Positive } \\
\text { gold standard }\end{array}$ & $\begin{array}{c}\text { Negative } \\
(N)\end{array}$ & $\begin{array}{c}\text { Total } \\
(N)\end{array}$ \\
\hline Positive (N) & 24 & 9 & 33 \\
Negative (N) & 11 & 102 & 113 \\
Total (N) & 35 & 111 & 146 \\
\hline
\end{tabular}

Interobserver agreement on review of PET/CT images for the detection of peritoneal carcinomatosis

There was high agreement between reviewers in interpretation of the PET/CT images as shown in Table 3. The Kappa coefficient for interobserver agreement was 0.91 (95\%CI 0.83-80.99) with only 5 disagreements for 146 cases. In 4 of these cases, the disagreement involved the localization of a pathological uptake in the peritoneal topography (ileocaeco-appendicular adenopathy in two cases, nodes of the Treitz angle for one and hepatic dome metastasis for one). In the other case, reviewers disagreed on the pathological nature of the non-hypermetabolic densifications of fat tissue following a recurrence on a hepatectomy slice.

Table 3 Peritoneal carcinomatosis diagnosis according to reviewers after review of PET/CT images

\begin{tabular}{|c|c|c|c|}
\hline \multirow[b]{2}{*}{ Reviewer 1} & \multicolumn{2}{|c|}{ Reviewer 2} & \multirow{2}{*}{$\begin{array}{c}\text { Total } \\
(N)\end{array}$} \\
\hline & $\begin{array}{l}\text { Positive } \\
\text { (N) }\end{array}$ & $\begin{array}{c}\text { Negative } \\
\text { (N) }\end{array}$ & \\
\hline Positive (N) & 32 & 4 & 36 \\
\hline Negative (N) & 1 & 109 & 110 \\
\hline Total (N) & 33 & 113 & 146 \\
\hline
\end{tabular}

Examination of signs indicative of $P C$

As shown in Table 4, focal uptake was observed for 33 patients (Figure 1), three of whom did not have a positive PC diagnosis. Five patients had PC without focal uptake. Among them, diffuse uptake was observed for two patients with a PC. Peri-hepatic curvilinear uptake was observed for four patients, three of whom had a PC (Figure 2). The straight line sign was observed for eight patients, seven of whom had a PC (Figure 3). Epiploic uptake was observed for four patients, all of whom had a PC (Figure 4). In the mucinous cases, the CT component was very important to show the epiploic nodes with low uptake (Figure 5).

Table 4 Presence of signs of peritoneal carcinomatosis as assessed by PET/CT imaging

\begin{tabular}{lcc}
\hline & $\begin{array}{c}\text { True positives } \\
\text { out of } 31 \text { TP } \\
\text { patients) }\end{array}$ & $\begin{array}{c}\text { False positives } \\
\text { (out of 4 FP patients) }\end{array}$ \\
\hline Focal uptake & 30 & 3 \\
$\begin{array}{l}\text { Diffuse uptake } \\
\begin{array}{l}\text { Perihepatic } \\
\text { curvilinear uptake }\end{array}\end{array}$ & 3 & 0 \\
$\begin{array}{l}\text { Epiploic uptake } \\
\text { Straight line sign }\end{array}$ & 4 & 0 \\
\hline
\end{tabular}

Extent of $P C$

Among 65 operated patients, 19 had PC. The median PCI was 3 (range: $1-13)$ when assessed by surgeons $(n=16)$ and 4 (range: $1-10)$ on PET/CT $(n=12)$. The areas most 


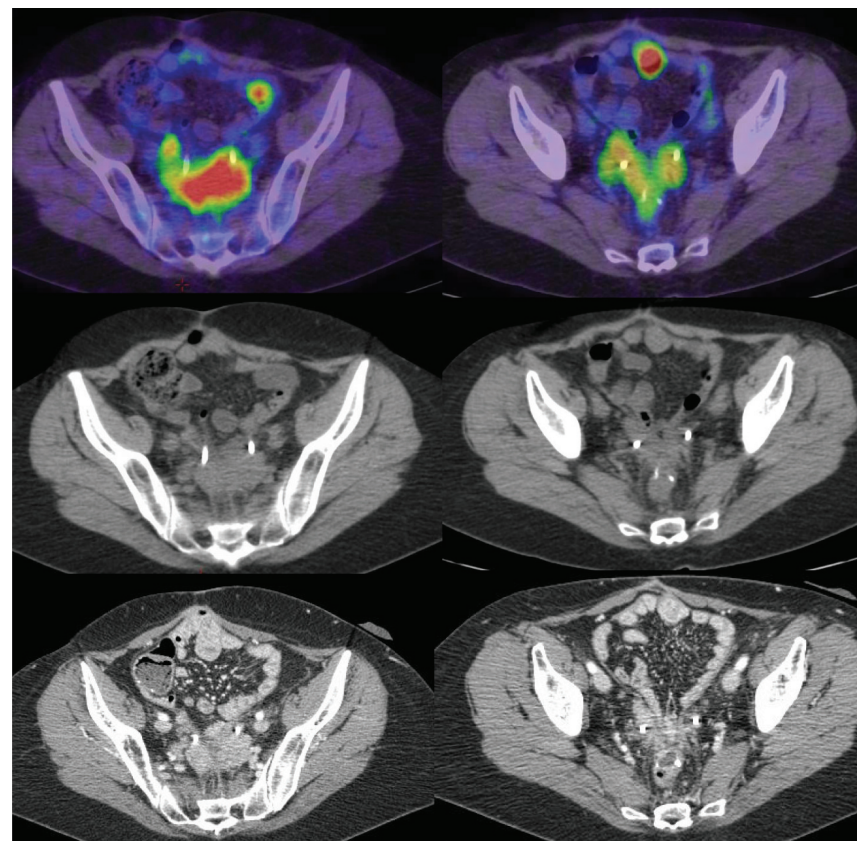

Figure 152 yo woman, fusion of the PET/CT (top), CT part of the PET/ CT (middle) and enhanced radiological CT (bottom) showing two smallintestinal focal uptakes without morphologic anomalies. It was two CP lesions, confirmed by pathology

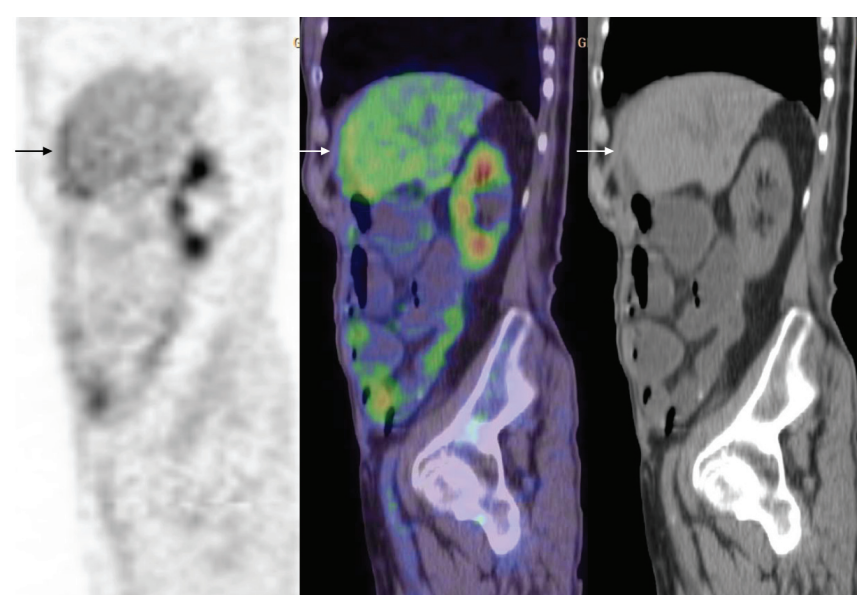

Figure 2 Curvilinear perihepatic uptake in a 75yo woman with CP extension (confirmed by radiological follow-up)

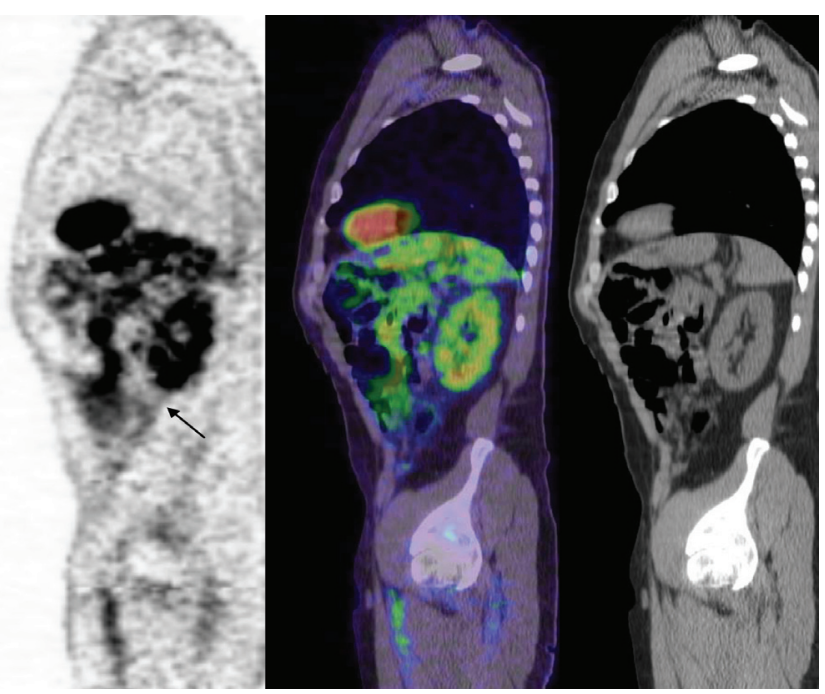

Figure 3 "Straight line sign" in a 45 yo male patient with PC extension confirmed by surgery

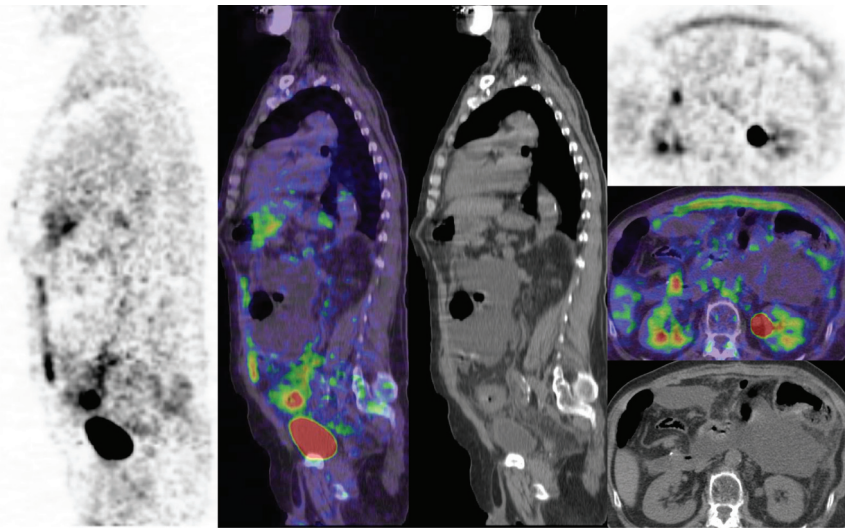

Figure 4 Epiploic thickening uptake in a 65yo male patient with $\mathrm{CP}$ extension confirmed by radiological follow up
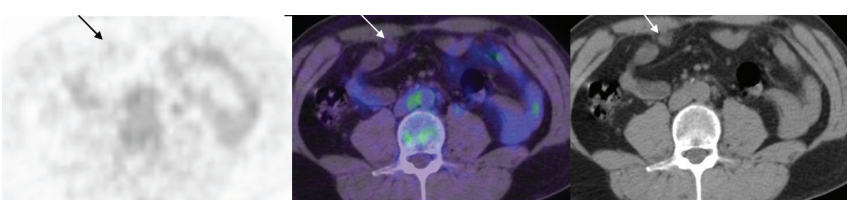

Figure 5 43yo man, epiploic node without significant uptake. After surgery, pathology showed CP node of mucinous adenocarcinoma

frequently involved were areas 1 and 6 (in $40 \%$ of cases), followed by area 0 (37.1\%), 5 (25.7\%), $3(22.8 \%)$ and 8 (22\%). Finally areas 7 and $4(17.1 \%)$, and 9 and $2(14.3 \%)$ were the least commonly involved (Figure 6).
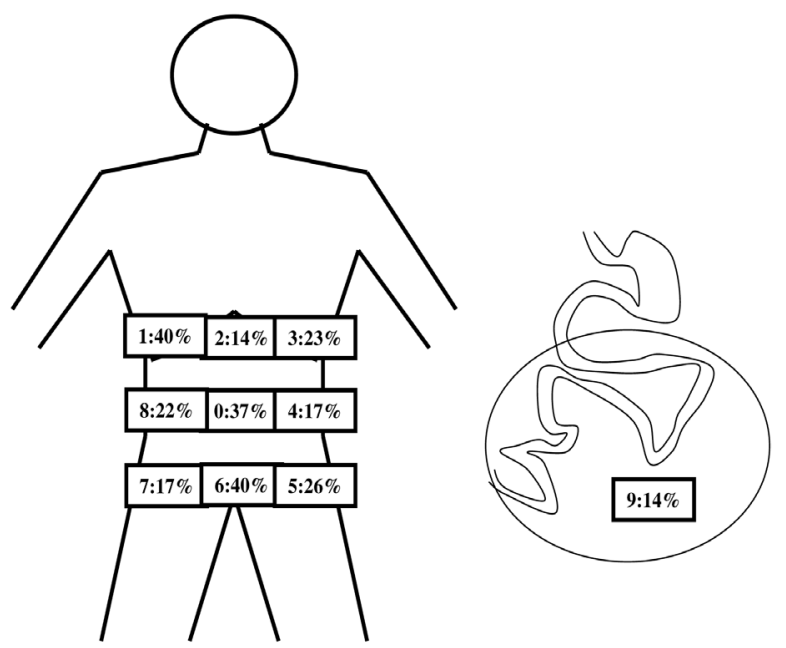

Figure 6 Areas of involvement of peritoneal carcinomatosis extent

\section{Discussion}

To our knowledge, this is the largest series to examine the identification of PC of CRC on a hybrid PET/CT camera, both in terms of patients $(n=146)$ and PC $(n=35)$. Previous series have included between 23 [12] and 107 [18] patients with 5 [12] to 40 [14] PC, but not all PC were of colorectal origin, possibly associated with various FDG uptake. The PET/CT method for CRC has been evaluated in larger series [19-25] but without a focus on PC. The prevalence of PC in our centre which is a regional reference centre for relapse cancer was $24 \%$ (35/146 patients). This rate is higher than those reported in the general colorectal 
cancer population [26], but of course lower than in series where suspicion of PC was an inclusion criterion [7-9].

The main finding is the high rate performance of PET/CT for PC detection with sensitivity of $88 \%$ and specificity of $96 \%$. These data are similar $[9,12,14,18]$ or higher than in previous studies $[7,10,11]$, irrespective of PET/CT hybrid camera use. To compare the performance of PET/ $\mathrm{CT}$ to $\mathrm{CT}$, an independent radiologist reviewed either the contemporary ceCT or the CT from the PET/CT (77/146). We judged this to be appropriate as the majority of anomalies we were looking for are found in abdominal fat, where a spontaneous contrast exists on CT images. However, identifying some PC lesions is facilitated by the injection of a vascular contrast product and the realisation of thin slices. In this study, diagnostic accuracy measures were not different according to type of CT used, with a lower rate of false positive in the non-enhanced CT from the PET/CT.

As in several published series, PET/CT demonstrated better diagnostic performance than the radiological CT $[9,11-14,25,27]$, but this is in contrast to as reported by Dromain et al. [7] who examined PC from various primary tumours (only 60\% colorectal cancer) and Lim et al. [10] who examined gastric tumours well known for lower FDG uptake. Further, methodological details were not given in either series for the review of the PET/CT images. A special reading of the CT component of PET/CT highlights the finding of small deposits and/or lesions with low uptake near the background.

The high inter observer agreement Kappa coefficient of 0.91 is higher than in another series where it has been reported at 0.46 [10]. This could be explained by the use of PET only in the other study, where reading is ambiguous due to lack of anatomical localisation, especially to separate physiological from neoplasic uptake (for example focal uptake in the ureter) or to localize neoplasic uptake either in the peritoneum or the retroperitoneum area. The second explanation is that we used preliminary training involving joint review of 20 patient files, which enabled us to define the different review stages and interpretation criteria. An independent review by a third of fourth nuclear physician would probably give a more realistic estimation. Four out of the five disagreements between observers were regarding whether the pathological uptake was peritoneal or not. The injection of an iodine contrast in the future would most definitely allow better identification of these cases.

As an exploratory analysis, we investigated the PC indices, but this was only available for $12 / 19$ patients with PC (of 65 with gold histological standard), and some discordances between PCI as indicated by the surgeon compared to on PET/CT were observed. Notably it seems that we may have overestimated the PC extent, once we discovered one sign.
In terms of signs, focal uptake was observed to be the principal indicator of $\mathrm{PC}$, found in $86 \%$ of PC cases. It should be noted that the 3 false positives for focal uptake corresponded to extra-peritoneal metastatic colorectal disease. Uptake significance was established visually and the focal uptake could be accompanied by underlying CT anomalies or not. A small-intestinal uptake, intense and well-differentiated could indicate a PC lesion even when no CT anomalies were visible (Figure 1). Our results highlight the focal and well-limited nature of the uptake in comparison to more poorly limited and more diffuse uptakes, which are compatible with physiological intestinal uptakes. Curvilinear peri-hepatic uptake, the straight line sign and epiploic uptake were relatively rare, but were good indicators of PC when present. The systematic searching for these signs was important, as they enabled us to diagnose PCs for 3 patients who had no focal uptake. The straight line sign was difficult to discern in slim patients with limited amounts of abdominal fat. This sign allows us to identify objectively a moderate and diffuse uptake of peritoneal fat by using retroperitoneal fat as a reference area. The presence of digestive structures especially on thin patient can imitate this sign which should only be sought between peritoneal and retroperitoneal fats. For these two signs, the 3D analyses in coronal and sagittal views remain important.

With regards to the mucinous histological subtype, we did not find any false negatives for the 20 patients who had a mucinous adenocarcinoma (9 of whom had a PC). However, a diagnosis was given on obvious CT anomalies without PET uptake (Figure 5). This may reflect the same phenomenon as described in a meta-analysis of studies using non-hybrid cameras [28], where sensitivity was lower than for mucinous adenocarcinomas.

The main difficulties encountered during review were to identify whether tumoral hypermetabolisms were of a peritoneal nature or not, particularly when they were situated at the pelvis, peri-hepatic or peri-colic levels or when they were close to nodal areas. The systematic utilisation of three series of images (PET only, CT only and PET/CT) enabled us to enhance review. The CT images from the PET/CT scans were not iodine contrast injected. The injection of the iodine product may have offered better identification, as demonstrated by Kitajima et al. [25].

The technical acquisition parameters that potentially influenced PET performance were under control in our series, eg. capillary glycaemia, activity injected and (18)F-FDG uptake delay were compliant with the Good Practice Guidelines [29]. The delay between the last chemotherapy cycle or last surgery before the PET/CT were also very good, with a median delay respectively of 7 months (minimum one month) and 14.5 months.

Given the retrospective nature of our series, the review was performed blindly to avoid maximum of bias. This 
prevented potential problems based on patient knowledge during functional imaging examinations, such as the discovery of a recent surgery during patient interviews which can modify imaging interpretation. Accordingly, we designed interpretation criteria to counter for these possible false positives and these appear to have been correctly identified in our data. The aspect of uptake with longitudinal linear contact with the abdominal wall was planned during the training readings which prevented false positives.

One of the main limitations of this study is that we only provide information on the presence of PC and limited data on the extent of PC $(n=12)$, which is essential for the surgeon to judge the complete resectability of the patient specifically the presence of extensive small bowel or mesentery involvement, segmental bowel obstructions, gross involvement of the hepato-gastric ligament, involvement of the liver hilus, presence of unresectable pelvic side wall involvement, obstruction of the ureters and involvement of the base of the urinary bladder.

Overall, the performances reported here should be compared to the performance of MRI with diffusion sequences [18, 30, 31]. However, in both the Satoh et al. [15] and Klumpp et al. [32] series, despite a heterogeneous population, PET/CT appears to be complementary to, or more accurate than, diffusion or DCE MRI [33]. Both techniques can underestimate the real extent of PC because of decreased sensitivity for lesions $<1 \mathrm{~cm}$ in size. Recent publications have successfully studied the extent of the small bowel involvement with CT-enteroclysis in the pre-operative setting in patients with peritoneal carcinomatosis of different primary origins requiring a nasojejunal catheter with rapid enteral administration of 1.8-2 L of enteral contrast medium [34].

\section{Conclusion}

(18)F-FDG PET/CT appears to be an accurate and reproducible technique for the diagnosis of peritoneal carcinomatosis of colorectal origin when specific PET and CT imaging interpretation methods are used. To confirm these results and to evaluate peritoneal carcinomatosis extension accurately, a prospective observational study using iodine contrast-enhanced PET/CT as a first imaging technique for pre-operative staging and potentially involving further independent reviewers remains to be undertaken.

\section{Funding disclosures}

No funding was received for this project.

\section{Conflicts of Interest}

The authors wish to express that they have no conflict of interest.

\section{Acknowledgements}

We would like to thank the Nuclear Medicine service at Hôpital Haut Leveque, Bordeaux for the use of their technical platform and staff, Jean Mendiboure for statistical help and Pippa McKelvie-Sebileau for medical writing services in English.

\section{References}

[1] Evrard S, Rivoire M, Arnaud J, Lermite E, Bellera C, et al. (2012) Unresectable colorectal cancer liver metastases treated by intraoperative radiofrequency ablation with or without resection. Br J Surg 99:558-565.

[2] Koppe MJ, Boerman OC, Oyen WJ, Bleichrodt RP (2006) Peritoneal carcinomatosis of colorectal origin: incidence and current treatment strategies. Ann Surg 243:212-222.

[3] Yang YY, Fleshman JW, Strasberg SM (2007) Detection and management of extrahepatic colorectal cancer in patients with resectable liver metastases. J Gastrointest Surg 11:929-944.

[4] Chua TC, Yan TD, Zhao J, Morris DL (2009) Peritoneal carcinomatosis and liver metastases from colorectal cancer treated with cytoreductive surgery perioperative intraperitoneal chemotherapy and liver resection. Eur J Surg Oncol 35:1299-1305.

[5] Kianmanesh R, Scaringi S, Sabate JM, Castel B, Pons-Kerjean N, et al. (2007) Iterative cytoreductive surgery associated with hyperthermic intraperitoneal chemotherapy for treatment of peritoneal carcinomatosis of colorectal origin with or without liver metastases. Ann Surg 245:597-603.

[6] Sugarbaker PH (1999) Management of peritoneal-surface malignancy: the surgeon's role. Langenbecks Arch Surg 384:576587.

[7] Dromain C, Leboulleux S, Auperin A, Goere D, Malka Det, et al. (2008) Staging of peritoneal carcinomatosis: enhanced CT vs. PET/ CT. Abdom Imaging 33:87-93.

[8] Pfannenberg C, Königsrainer I, Aschoff P, Oksüz MO, Zieker D, et al. (2009) (18)F-FDG-PET/CT to select patients with peritoneal carcinomatosis for cytoreductive surgery and hyperthermic intraperitoneal chemotherapy. Ann Surg Oncol 16:1295-1303.

[9] Dirisamer A, Schima W, Heinisch M, Weber M, Lehner HP, et al. (2009) Detection of histologically proven peritoneal carcinomatosis with fused 18F-FDG-PET/MDCT. Eur J Radiol 69:536-541.

[10] Lim JS, Kim MJ, Yun MJ, Oh YT, Kim JH, et al. (2006) Comparison of CT and 18F-FDG pet for detecting peritoneal metastasis on the preoperative evaluation for gastric carcinoma. Korean J Radiol 7:249-256.

[11] Suzuki A, Kawano T, Takahashi N, Lee J, Nakagami Y, et al. (2004) Value of 18F-FDG PET in the detection of peritoneal carcinomatosis. Eur J Nucl Med Mol Imaging 31:1413-1420.

[12] Tanaka T, Kawai Y, Kanai M, Taki Y, Nakamoto Y, et al. (2002) Usefulness of FDG-positron emission tomography in diagnosing peritoneal recurrence of colorectal cancer. Am J Surg 184:433436.

[13] Turlakow A, Yeung HW, Salmon AS, Macapinlac HA, Larson SM (2009) Peritoneal carcinomatosis: role of (18)F-FDG PET. J Nucl Med 44:1407-1412.

[14] Zhang M, Jiang X, Zhang M, Xu H, Zhai G, et al. (2009) The Role of 18F-FDG PET/CT in the evaluation of Ascites of Undetermined Origin. J Nucl Med 50:506-512.

[15] Gupta P (2006) The straight line sign. Radiology 240:611-612.

[16] Lin EC (2002) Straight line sign of diffuse peritoneal carcinomatosis on sagittal FDG positron emission tomographic images. Clin Nucl Med 27:735-736.

[17] Véron-Piot S CS, Diebold MD, Avisse C, Marcus C (2007) Peritoneal carcinomatosis: what should be done, how, when and where? Feuillets de radiologie 47:260-268.

[18] Satoh Y, Ichikawa T, Motosugi U, Kimura K, Sou H, et al. (2011) Diagnosis of peritoneal dissemination: comparison of 18F-FDG PET/CT, diffusion-weighted MRI, and contrast-enhanced MDCT. AJR Am J Roentgenol 196:447-453.

[19] de Geus-Oei LF, Wiering B, Krabbe PF, Ruers TJ, Punt CJ, et al. (2006) FDG-PET for prediction of survival of patients with metastatic colorectal carcinoma. Ann Oncol 17:1650-1655.

[20] Ruers TJ, Wiering B, van der Sijp JR, Roumen RM, de Jong KP, et al. (2009) Improved selection of patients for hepatic surgery of colorectal liver metastases with (18)F-FDG PET: a randomized study. J Nucl Med 50:1036-1041. 
[21] Scott AM, Gunawardana DH, Kelley B, Stuckey JG, Byrne AJ, et al. (2008) PET changes management and improves prognostic stratification in patients with recurrent colorectal cancer: results of a multicenter prospective study. J Nucl Med 49:1451-1457.

[22] Valk PE, Abella-Columna E, Haseman MK, Pounds TR, Tesar RD, et al.(1999) Whole-body PET imaging with [18F]fluorodeoxyglucose in management of recurrent colorectal cancer. Arch Surg 134:503511.

[23] Wiering B, Krabbe PF, Dekker HM, Oyen WJ, Ruers TJ (2007) The role of FDG-PET in the selection of patients with colorectal liver metastases. Ann Surg Oncol 14:771-779.

[24] Zervos EE, Badgwell BD, Burak WEJr, Arnold MW, Martin EW (2001) Fluorodeoxyglucose positron emission tomography as an adjunct to carcinoembryonic antigen in the management of patients with presumed recurrent colorectal cancer and nondiagnostic radiologic workup. Surgery 130:636-643.

[25] Kitajima K, Murakami K, Yamasaki E, Domeki Y, Tsubaki M, et al. (2009) Performance of integrated FDG PET/contrast-enhanced CT in the diagnosis of recurrent colorectal cancer: Comparison with integrated FDG PET/non-contrast-enhanced CT and enhanced CT. Eur J Nucl Med Mol Imaging 3:1388-1396.

[26] Jayne DG, Fook S, Loi C, Seow-Choen F (2002) Peritoneal carcinomatosis from colorectal cancer. Br J Surg 89:1545-1550.

[27] Wang PH, Liu RS, Li YF, Ng HT, Yuan CC (2000) Whole-body PET with (fluorine-18)-2-deoxyglucose for detecting recurrent primary serous peritoneal carcinoma: An initial report. Gynecol Oncol 77:44-47.

[28] Whiteford MH, Whiteford HM, Yee LF, Ogunbiyi OA, Dehdashti F, et al. (2000) Usefulness of FDG-PET scan in the assessment of suspected metastatic or recurrent adenocarcinoma of the colon and rectum. Dis Colon Rectum 43:759-767; discussion 67-70

[29] Monteil J, Jocob TH, Vandroux JC, Mundler O (2004) Réalisation pratique d'un examen TEP-TDM: de l'indication au compte rendu. Médecine Nucléaire-Imagerie fonctionnelle et métabolique 28:189203.

[30] Fujii S, Matsusue E, Kanasaki Y, Kanamori Y, Nakanishi J, et al. (2008) Detection of peritoneal dissemination in gynecological malignancy: evaluation by diffusion-weighted MR imaging. Eur Radiol 18:1823.

[31] Tempany CM, Zou KH, Silverman SG, Brown DL, Kurtz AB, et al. (2008) Staging of advanced ovarian cancer: comparison of imaging modalities--report from the Radiological Diagnostic Oncology Group. Radiology 215:761-767.

[32] Klumpp BD, Schwenzer N, Aschoff P, Miller S, Kramer U, et al. (2013) Preoperative assessment of peritoneal carcinomatosis: intraindividual comparison of 18F-FDG PET/CT and MRI. Abdom Imaging 38:64-71.

[33] Soussan M, Des Guetz G, Barrau V, Aflalo-Hazan V, Pop G, et al. (2012) Comparison of FDG-PET/CT and MR with diffusionweighted imaging for assessing peritoneal carcinomatosis from gastrointestinal malignancy. Eur Radiol 22:1479-1487.

[34] Courcoutsakis N, Tentes AA, Astrinakis E, Zezos P, Prassopoulos P (2013) CT-Enteroclysis in the preoperative assessment of the smallbowel involvement in patients with peritoneal carcinomatosis, candidates for cytoreductive surgery and hyperthermic intraperitoneal chemotherapy. Abdom Imaging. 38:56-63. 